\title{
Constraints on Light Neutrino Parameters Derived from the Study of Neutrinoless Double Beta Decay
}

\author{
Sabin Stoica ${ }^{1,2}$ and Andrei Neacsu ${ }^{1,2}$ \\ ${ }^{1}$ Horia Hulubei Foundation, P.O. Box MG-12, Romania \\ ${ }^{2}$ Horia Hulubei National Institute of Physics and Nuclear Engineering, P.O. Box MG-6, 077125 Magurele, Bucharest, Romania
}

Correspondence should be addressed to Sabin Stoica; stoica@theory.nipne.ro

Received 7 February 2014; Accepted 28 April 2014; Published 28 May 2014

Academic Editor: Abhijit Samanta

Copyright (C) 2014 S. Stoica and A. Neacsu. This is an open access article distributed under the Creative Commons Attribution License, which permits unrestricted use, distribution, and reproduction in any medium, provided the original work is properly cited. The publication of this article was funded by SCOAP S $^{3}$

The study of the neutrinoless double beta $(0 \nu \beta \beta)$ decay mode can provide us with important information on the neutrino properties, particularly on the electron neutrino absolute mass. In this work we revise the present constraints on the neutrino mass parameters derived from the $0 \nu \beta \beta$ decay analysis of the experimentally interesting nuclei. We use the latest results for the phase space factors (PSFs) and nuclear matrix elements (NMEs), as well as for the experimental lifetime limits. For the PSFs we use values computed with an improved method reported very recently. For the NMEs we use values chosen from the literature on a case-by-case basis, taking advantage of the consensus reached by the community on several nuclear ingredients used in their calculation. Thus, we try to restrict the range of spread of the NME values calculated with different methods and, hence, to reduce the uncertainty in deriving limits for the Majorana neutrino mass parameter. Our results may be useful to have an updated image on the present neutrino mass sensitivities associated with $0 v \beta \beta$ measurements for different isotopes and to better estimate the range of values of the neutrino masses that can be explored in the future double beta decay (DBD) experiments.

\section{Introduction}

Neutrinoless double beta decay is a beyond standard model (BSM) process by which an even-even nucleus transforms into another even-even nucleus with the emission of two electrons/positrons but no antineutrinos/neutrinos in the final states. Its study is very attractive because it would clarify the question about the lepton number conservation, decide on the neutrinos character (are they Dirac or Majorana particles?), and give a hint on the scale of their absolute masses. Moreover, the study of the $0 \nu \beta \beta$ decay has a broader potential to search for other BSM phenomena. The reader can find up-to-date information on these studies from several recent reviews [1-6], which also contain therein a comprehensive list of references in the domain.

The scale of the absolute mass of neutrinos is a key issue for understanding the neutrino properties. It cannot be derived from neutrino oscillation experiments which can only measure the square of the neutrino mass differences between different flavors [7-12]. Analysis of $0 \nu \beta \beta$ decay and cosmological data are at present the most sensitive ways to investigate this issue.

The lifetime of the $0 \nu \beta \beta$ decay modes can be expressed, in a good approximation, as a product of a phase space factor (depending on the atomic charge and energy released in the decay, $Q_{\beta \beta}$ ), a nuclear matrix element (related to the nuclear structure of the parent and daughter nuclei), and a lepton number violation (LNV) parameter (related to the BSM mechanism considered). Thus, to extract reliable limits for the LNV parameters we need accurate calculations of both PSFs and NMEs, as well as reliable measurements of the lifetime.

The largest uncertainties in theoretical calculations for DBD are related to the NMEs values. That is why there is a continuous effort in the literature to develop improved nuclear structure methods for their computation. At present, the NMEs are computed by several methods which differ conceptually, the most employed being the protonneutron quasi-random phase approximation (pnQRPA) [1320], interacting shell model (ISM) [21-25], interacting 
boson approximation (IBA) [26-28], projected HartreeFock-Bogoliubov (PHFB) [29, 30], and energy density functional (EDS) method [31]. There are still large differences between the NMEs values computed with different methods and by different groups, and these have been largely discussed in the literature (see, e.g., $[2,3]$ ). On the other side, there is a consensus in the community on the way that several nuclear effects and nuclear parameters should be used in calculations. In this work, we take advantage of this consensus when we chose the NMEs values, trying to restrict their range of spread and, consequently, to reduce the uncertainty in deriving the neutrino Majorana mass parameters.

Unlike the NMEs, the PSFs have been calculated a long time ago [32-39] and were considered to be computed with enough precision. However, recently, they were recalculated within an improved approach, by using exact electron Dirac wave functions (w.f.) taking into account the finite nuclear size and electron screening effects [40]. The authors found differences between their results and those calculated previously with approximate electron w.f., especially for heavier nuclei. We have also independently recalculated the PSFs by developing new routines for computing the relativistic (Dirac) electron w.f. by taking into account the nuclear finite size and screening effects. In addition, we use a Coulomb potential derived from a realistic proton density distribution in the daughter nucleus $[41,42]$. In this work we use new PSFs values obtained by improving the numerical precision of our routines as compared with our previous works. The obtained values are very close to those reported in $[40,41]$.

Finally, for the lifetime limits, we take the most recent results found in the literature.

The paper is organized as follows. In the next section we shortly recall the general formalism for the derivation of the neutrino mass parameters from $0 \nu \beta \beta$ decay analysis, highlighting the nuclear ingredients involved in calculations. In Section 3 we discuss the way of choosing the NME values and report our results for the light neutrino Majorana mass parameters, while in Section 4 we formulate the conclusions of our work.

\section{Formalism}

We shortly present the general formalism for the derivation of neutrino mass parameters from $0 \nu \beta \beta$ decay analysis. We start with the lifetime formula and then describe the main steps and ingredients used in the theoretical calculation of their components, that is, PSFs and NMEs.

Assuming that the dominant mechanism of occurrence for the $0 \nu \beta \beta$ decay mode is the exchange of Majorana lefthanded light neutrinos between two nucleons from the parent nucleus, the lifetime reads

$$
\left(T_{1 / 2}^{0 v}\right)^{-1}=G^{0 v}\left(Q_{\beta \beta}, Z\right)\left|M^{0 v}\right|^{2}\left(\frac{\left\langle m_{\nu}\right\rangle}{m_{e}}\right)^{2},
$$

where $G^{0 v}$ are the PSFs for this decay mode, depending on the energy decay $Q_{\beta \beta}$ and nuclear charge $Z, M^{0 v}$ are the corresponding NMEs, depending on the nuclear structure of the parent and daughter nuclei involved in the decay, $m_{e}$ is electron mass, and $\left\langle m_{\nu}\right\rangle$ is the light neutrino Majorana mass parameter. This parameter can be expressed as a (coherent) linear combination of the light neutrino masses:

$$
\left\langle m_{\nu}\right\rangle=\left|\sum_{k=1}^{3} U_{e k}^{2} m_{k}\right|,
$$

where $U_{e k}$ are the elements of the first row in the PMNS (Pontecorvo-Maki-Nakagawa-Sakata) neutrino matrix and $m_{k}$ are the light neutrino masses [56]. From (1) the expression of $m_{v}$ reads

$$
\left\langle m_{\nu}\right\rangle=\frac{m_{e}}{\left|M^{0 v}\right| \sqrt{T^{0 v} \cdot G^{0 v}}} .
$$

For deriving $\left\langle m_{\nu}\right\rangle$ we need accurate calculations of both PSFs and NMEs for each isotope for which there are experimental lifetime limits. The PSFs have been calculated a long time ago in some approximations [32-39] and were considered, until recently, to be computed with enough precision. However, they were recalculated recently in [4042] using more advanced approaches for the numerical evaluation of the Dirac wave functions with the inclusion of nuclear finite size and screening effects. In addition, in [41] the usual Coulomb spherical potential was replaced by another one, derived from a more realistic proton density distribution in the daughter nucleus. These recent PSF calculations led to significant differences in comparison to the older calculations, especially for the heavier isotopes, that should be taken into account for a precise derivation of the neutrino mass parameters.

The computation of the NMEs is a subject of debate in the literature for long time, because they bring the large uncertainties in the theoretical calculations for DBD. Different groups have developed several conceptually different nuclear structure methods [13-31], as we have mentioned in the previous section. The expression of the NMEs can be written, in general, as a sum of three components:

$$
M^{0 v}=M_{\mathrm{GT}}^{0 v}-\left(\frac{g_{V}}{g_{A}}\right)^{2} \cdot M_{F}^{0 v}-M_{T}^{0 v},
$$

where $M_{\mathrm{GT}}^{0 \nu}, M_{F}^{0 v}$, and $M_{T}^{0 v}$ are the Gamow-Teller (GT), Fermi $(F)$, and Tensor $(T)$ components, respectively. These are defined as follows:

$$
M_{\alpha}^{0 v}=\sum_{m, n}\left\langle 0_{f}^{+}\left\|\tau_{-m} \tau_{-n} O_{m n}^{\alpha}\right\| 0_{i}^{+}\right\rangle,
$$

where $O_{m n}^{\alpha}$ are transition operators $(\alpha=\mathrm{GT}, F, T)$ and the summation is performed over all the nucleon states. An important part of the NME calculation is the computation of the reduced matrix elements of the two-body transition operators $O^{\alpha}$. Their calculation can be decomposed into products of reduced matrix elements within the spin and relative coordinate spaces. Their explicit expressions are [4, 23]

$$
\begin{aligned}
& O_{12}^{\mathrm{GT}}=\sigma_{1} \cdot \sigma_{2} H(r), \quad O_{12}^{F}=H(r), \\
& O_{12}^{T}=\sqrt{\frac{2}{3}}\left[\sigma_{1} \times \sigma_{2}\right]^{2} \cdot \frac{r}{R} H(r) C^{(2)}(\widehat{r}) .
\end{aligned}
$$


The most difficult is the computation of the radial part of the two-body transition operators, which contains the neutrino potentials. These potentials depend weakly on the intermediate states and are defined by integrals of momentum carried by the virtual neutrino exchanged between the two nucleons [16]:

$$
\begin{aligned}
H_{\alpha}(r) & =\frac{2 R}{\pi} \int_{0}^{\infty} j_{i}(q r) \frac{h_{\alpha}(q)}{\omega} \frac{1}{\omega+\langle E\rangle} q^{2} d q \\
& \equiv \int_{0}^{\infty} j_{i}(q r) V_{\alpha}(q) q^{2} d q,
\end{aligned}
$$

where $R=r_{0} A^{1 / 3} \mathrm{fm}\left(r_{0}=1.2 \mathrm{fm}\right), \omega=\sqrt{q^{2}+m_{v}^{2}}$ is the neutrino energy, and $j_{i}(q r)$ is the spherical Bessel function ( $i=0,0$, and 2 for GT, $F$, and $T$, resp.). Usually, in calculations one uses the closure approximation which consists of a replacement of the excitation energies of the states in the intermediate odd-odd nucleus contributing to the decay, by an average expression $\langle E\rangle$. This approximation works well in the case of $0 \nu \beta \beta$ decay modes and simplifies much the calculations. The expressions of $h_{\alpha}(\alpha=\mathrm{GT}, F, T)$ are

$$
\begin{aligned}
& h_{F}=G_{V}^{2}\left(q^{2}\right), \\
& h_{\mathrm{GT}}\left(q^{2}\right)= \frac{G_{A}^{2}\left(q^{2}\right)}{g_{A}^{2}}\left[1-\frac{2}{3} \frac{q^{2}}{q^{2}+m_{\pi}^{2}}+\frac{1}{3}\left(\frac{q^{2}}{q^{2}+m_{\pi}^{2}}\right)^{2}\right] \\
&+\frac{2}{3} \frac{G_{M}^{2}\left(q^{2}\right)}{g_{A}^{2}} \frac{q^{2}}{4 m_{p}^{2}}, \\
& h_{T}\left(q^{2}\right)= \frac{G_{A}^{2}\left(q^{2}\right)}{g_{A}^{2}}\left[\frac{2}{3} \frac{q^{2}}{q^{2}+m_{\pi}^{2}}-\frac{1}{3}\left(\frac{q^{2}}{q^{2}+m_{\pi}^{2}}\right)^{2}\right] \\
&+\frac{1}{3} \frac{G_{M}^{2}\left(q^{2}\right)}{g_{A}^{2}} \frac{q^{2}}{4 m_{p}^{2}},
\end{aligned}
$$

where $m_{\pi}$ is the pion mass, $m_{p}$ is the proton mass, and

$$
G_{M}\left(q^{2}\right)=\left(\mu_{p}-\mu_{n}\right) G_{V}\left(q^{2}\right)
$$

with $\left(\mu_{p}-\mu_{n}\right)=4.71$.

The expressions (9)-(10) include important nuclear ingredients that should be taken into account for a precise computation of the NMEs, such as the higher order currents in the nuclear interaction (HOC) and finite nucleon size effect (FNS). Inclusion of HOC brings additional terms in the $H_{\mathrm{GT}}$ component and leads to the appearance of the $H_{T}$ component in the expressions of the neutrino potentials. FNS effect is taken into account through $G_{V}$ and $G_{A}$ form factors:

$$
\begin{aligned}
& G_{A}\left(q^{2}\right)=g_{A}\left(\frac{\Lambda_{A}^{2}}{\Lambda_{A}^{2}+q^{2}}\right)^{2}, \\
& G_{V}\left(q^{2}\right)=g_{V}\left(\frac{\Lambda_{V}^{2}}{\Lambda_{V}^{2}+q^{2}}\right)^{2} .
\end{aligned}
$$

For the vector and axial coupling constants, the majority of the calculations take either the quenched value, $g_{V}=1$, or the unquenched one, $g_{A}=1.25$, while the values of the vector and axial vector form factors are $\Lambda_{V}=850 \mathrm{MeV}$ and $\Lambda_{A}=1086 \mathrm{MeV}$ [1], respectively. As one can see, when HOC and FNS corrections are included in the calculations, the dependence of NMEs expression on $g_{A}$ is not trivial and the NMEs values obtained with the quenched or the unquenched value of this parameter cannot be obtained by simply rescaling.

To compute the radial matrix elements $\left\langle n l\left|H_{\alpha}\right| n^{\prime} l^{\prime}\right\rangle$ an important ingredient is the adequate inclusion of SRCs, induced by the nuclear interaction. The way of introducing the SRC effects has also been subject of debate $([16-18,20])$. The SRC effects are included by correcting the single particle w.f. as follows:

$$
\psi_{n l}(r) \longrightarrow[1+f(r)] \psi_{n l}(r) .
$$

The correlation function $f(r)$ can be parametrized in several ways. There are three parameterizations which are used, Miller-Spencer (MS), UCOM, and CCM (with CD-Bonn and AV18 potentials). The Jastrow prescription [34] for the correlation function is

$$
f(r)=-c \cdot e^{-a r^{2}}\left(1-b r^{2}\right)
$$

and includes all these parameterizations, depending on values of the $a, b, c$ parameters.

Including HOC and FNS effects, the radial matrix elements of the neutrino potentials become

$$
\begin{aligned}
\left\langle n l\left|H_{\alpha}(r)\right| n^{\prime} l^{\prime}\right\rangle= & \int_{0}^{\infty} r^{2} d r \psi_{n l}(r) \psi_{n^{\prime} l^{\prime}}(r)[1+f(r)]^{2} \\
& \times \int_{0}^{\infty} q^{2} d q V_{\alpha}(q) j_{n}(q r)
\end{aligned}
$$

where $v$ is the oscillator constant and $V_{\alpha}(q)$ is an expression containing the $q$ dependence of the neutrino potentials.

From (4)-(15) one can see that a set of approximations and parameters are involved in the NMEs expressions, as the HOC, FNS, and SRC effects and $g_{A}, r_{0},\left(\Lambda_{A}, \Lambda_{B}\right),\langle E\rangle$ parameters. Are there any recommendations on how should they be included in the calculations? At present there is a general consensus in the community in this respect that will be discussed in the next section.

\section{Numerical Results and Discussions}

The neutrino mass parameters are derived from (3). To get $\left\langle m_{\nu}\right\rangle$ in the same units as $m_{e}$ we take the NMEs dimensionless and the PSFs $\left(G^{0 \nu}\right)$ in units of $[\mathrm{yr}]^{-1}$.

The PSF values were obtained by recalculating them with our code, developed in [41], but with improved numerical precision. At this point we mention that the improved PSF values come, on the one hand, by the use of a Coulomb potential describing a more realistic proton charge density in the daughter nucleus instead of the (usual) constant charge density one, to solve the Dirac equations for obtaining the 
electron w.f. On the other hand, we got better precision of our numerical routines that compute the PSFs by enhancing the number of the interpolation points on a case-to-case basis until the results become stationary. The obtained values are very close to both those reported previously in [40,41]. This gives us confidence on their reliability. We mention that these PSFs values differ from older calculations as, for example, those reported in $[33,35-37]$ by up to $28 \%$. Such differences are important for precise estimations and justify the reactualization of the PSFs values in extracting Majorana neutrino mass parameters.

For the experimental lifetime we took the most recent results reported in the literature. In particular, we remark the newest results for ${ }^{76} \mathrm{Ge}$ from GERDA [49] and for ${ }^{136} \mathrm{Xe}$ from KamLAND-Zen [54].

The largest uncertainty in the derivation of $\left\langle m_{\nu}\right\rangle$ comes from the values of the NMEs. Fortunately, at present there is a general consensus in the community on the employment of the different nuclear effects (approximations) and parameters which appear in the NMEs expressions (see (4)-(15)) [57]. Thus, one can restrict the range of spread of the NMEs values for a particular nucleus, if one takes into account some recommendations resulting from the analysis of many NMEs calculations. For example, one recommends the inclusion in calculation of the HOC, FNS, and SRC effects (although their effects can partially compensate each other [43]). For SRCs, softer parametrizations like UCOM $[17,18,20]$ and CCM [5860] are recommended, while the MS produces a too severe cut of the w.f. for very short internucleon distances, which reflects into smaller NMEs values. Concerning the nuclear parameters, one recommends the use of an unquenched value for the $g_{A}$ axial vector constant, the values specified above for the vector and axial vector form factors $\left(\Lambda_{V}, \Lambda_{A}\right)$, and a value of $r_{0}=1.2 \mathrm{fm}$ for the nuclear radius constant. The value for the average energy $(\langle E\rangle)$, used in the closure approximation, is a function of atomic mass $A$, but the results are less sensitive to changes within a few $\mathrm{MeV}$. The use in different ways of these ingredients can result in significant differences between the NMEs values. Hence, a consensus is useful to approach the results obtained by different groups. Having agreement on these nuclear ingredients, the differences in the NMEs values should be searched in the features of the different nuclear structure methods. These methods use different ways of building the wave functions and different specific model spaces and type of nucleon-nucleon correlations and use some specific parameters $[3,24,43]$. Unfortunately, the uncertainties in the NMEs calculation associated with a particular nuclear structure method cannot be easily fixed and they are still a subject of debate. As a general feature, ShM calculations underestimate the NMEs values (due to the limitations of the model spaces used), while the other methods overestimate them. There are, however, a few hints on how to understand/bring closer the NMEs results obtained with different methods. One idea would be to analyze the structure of the wave functions used in terms of the seniority scheme [57]. Another one is to (re)calculate the NME values as to reproduce s.p. occupancies numbers measured recently for ${ }^{76} \mathrm{Ge}$ and ${ }^{82} \mathrm{Se}$ nuclei [61, 62]. For example, when the
QRPA calculations have been modified with the s.p. energies that reproduce the experimental occupancies, the new QRPA NMEs values are much closer to the ShM ones.

In Table 1 we display the NMEs values obtained with different nuclear methods. For uniformity and in agreement with the consensus discussed above, we chose those results that were performed with the inclusion of HOC, FNS, and SRC (UCOM and CD-Bonn) effects and with unquenched $g_{A}=1.25$, as nuclear ingredients. We mention that the newest experimental determinations of this parameter report values even larger $(1.269,1.273)$ [63]. However, the differences between NMEs values obtained with $g_{A}=1.25-$ 1.273 are not significant [43]. The NMEs values for ${ }^{76} \mathrm{Ge}$ and ${ }^{82} \mathrm{Se}$ written in parenthesis represent the adjusted NMEs values obtained with QRPA method by the Tuebingen and Jyvaskyla groups, when the s.p. energies were adjusted to the occupancy numbers reported in $[61,62]$. One remarks that the QRPA calculations with s.p. occupancies in accordance with experiment get significantly close to the ShM results, which is remarkable. In the future, one expects measurements of the occupancy numbers for other nuclei, as well. Also, it would be interesting if other methods, besides QRPA, would try to recalculate the NMEs by adjusting s.p. energies to experimental occupancy numbers.

We also make some remarks about the NMEs values on a case-by-case basis. For ${ }^{48} \mathrm{Ca}$ we appreciate that ShM calculations give more realistic results than the other methods. In support of this claim we mention that, in the case of this isotope, ShM calculations are performed within the full pf shell and using good effective $\mathrm{NN}$ interactions, checked experimentally on other spectroscopic quantities $[43,44$, 64]. Also, we remark that NMEs values obtained with ShM for this isotope were used to correctly predict $T_{1 / 2}^{2 \nu}$, before its experimental measurement [65]. For the isotopes with $A=96-130$ there is a larger spread of the NMEs values calculated with different methods and, consequently, a larger uncertainty in predicting the $\left\langle m_{\nu}\right\rangle$ parameters. For ${ }^{136} \mathrm{Xe}$ there are new ShM large-scale calculations with inclusion of a larger model space than the older calculations [45]. For this isotope the NMEs values are more grouped. Corroborated with a quite good experimental lifetime, from this isotope one gets the most stringent constraint for the $\left\langle m_{\nu}\right\rangle$ parameter.

In Table 2 we present our results for the Majorana neutrino mass parameters $\left(\left\langle m_{\nu}\right\rangle\right)$ together with the values of $Q_{\beta \beta}$, the PSFs $\left(G^{0 \nu}\right)$, NMEs $\left(M^{0 \nu}\right)$, and experimental lifetime $\left(T_{1 / 2}^{0 \nu}\right)$ for all the isotopes for which data exists. Making a sort of the NMEs values from the literature according to the considerations presented, we reduce the interval of their spread to about a factor of 2 , even less (with one exception). This results in reducing the uncertainty in deriving the constraints on the light neutrino Majorana mass parameters, while taking into account NME values obtained with all the main nuclear methods existent on the market. One observes that the stringent constraints are obtained from the ${ }^{136} \mathrm{Xe}$ isotope, followed by the ${ }^{76} \mathrm{Ge}$ one. This is due to both the experimental sensitivity of the experiments measuring these isotopes and the reliability of the PSFs and NMEs theoretical calculations. The experiments measuring these 
TABle 1: The NMEs obtained with different methods. The values are obtained using an unquenched value for $g_{A}$ and softer SRC parametrizations, which are specified in the second column.

\begin{tabular}{|c|c|c|c|c|c|c|c|c|c|c|}
\hline Method & SRC & ${ }^{48} \mathrm{Ca}$ & ${ }^{76} \mathrm{Ge}$ & ${ }^{82} \mathrm{Se}$ & ${ }^{96} \mathrm{Zr}$ & ${ }^{100} \mathrm{Mo}$ & ${ }^{116} \mathrm{Cd}$ & ${ }^{130} \mathrm{Te}$ & ${ }^{136} \mathrm{Xe}$ & ${ }^{150} \mathrm{Nd}$ \\
\hline [43] ShM & CD-BONN & 0.81 & 3.13 & 2.88 & & & & & & \\
\hline [44] ShM & CD-BONN & 0.90 & & & & & & & $2.21[45]$ & \\
\hline [24] ShM & UCOM & 0.85 & $2.81-3.52$ & 2.64 & & & & 2.65 & 2.19 & \\
\hline [27] IBM-2 & CD-BONN & 2.38 & 6.16 & 4.99 & 3.00 & 4.50 & 3.29 & 4.61 & 3.79 & 2.88 \\
\hline [3] QRPA & CD-BONN & & $5.93(3.27)$ & $5.30(4.54)$ & 2.19 & 4.67 & 3.72 & 4.80 & 3.00 & $3.16[46]$ \\
\hline [47] QRPA & UCOM & & $5.36(4.11)$ & 3.72 & 3.12 & 3.93 & 4.79 & 4.22 & 2.80 & \\
\hline [31] GCM & CD-BONN & 2.37 & 4.60 & 4.22 & 5.65 & 5.08 & 4.72 & 5.13 & 4.20 & 1.71 \\
\hline$[29,30]$ PHFB & CD-BONN & & & & 2.98 & 6.07 & & 3.98 & & 2.68 \\
\hline
\end{tabular}

TABLe 2: Majorana neutrino mass parameters together with the other components of the $0 v \beta \beta$ decay halftimes: the $Q_{\beta \beta}$ values, the experimental lifetime limits, the phase space factors, and the nuclear matrix elements.

\begin{tabular}{|c|c|c|c|c|c|}
\hline & $Q_{\beta \beta}[\mathrm{MeV}]$ & $T_{\exp }^{0 \nu \beta \beta}[\mathrm{yr}]$ & $G^{0 \nu \beta \beta}\left[\mathrm{yr}^{-1}\right]$ & $M^{0 \nu \beta \beta}$ & $\left\langle m_{\nu}\right\rangle[\mathrm{eV}]$ \\
\hline${ }^{48} \mathrm{Ca}$ & 4.272 & $>5.810^{22}[48]$ & $2.46 E-14$ & $0.81-0.90$ & $<[15.0-16.7]$ \\
\hline${ }^{76} \mathrm{Ge}$ & 2.039 & $>2.110^{25}[49]$ & $2.37 E-15$ & $2.81-6.16$ & $<[0.37-0.82]$ \\
\hline${ }^{82} \mathrm{Se}$ & 2.995 & $>3.610^{23}[50]$ & $1.01 E-14$ & $2.64-4.99$ & $<[1.70-3.21]$ \\
\hline${ }^{96} \mathrm{Zr}$ & 3.350 & $>9.210^{21}[51]$ & $2.05 E-14$ & $2.19-5.65$ & $<[6.59-17.0]$ \\
\hline${ }^{100} \mathrm{Mo}$ & 3.034 & $>1.110^{24}[50]$ & $1.57 E-14$ & $3.93-6.07$ & $<[0.64-0.99]$ \\
\hline${ }^{116} \mathrm{Cd}$ & 2.814 & $>1.710^{23}[52]$ & $1.66 E-14$ & $3.29-4.79$ & $<[2.00-2.92]$ \\
\hline${ }^{130} \mathrm{Te}$ & 2.527 & $>2.810^{24}[53]$ & $1.41 E-14$ & $2.65-5.13$ & $<[0.50-0.97]$ \\
\hline${ }^{136} \mathrm{Xe}$ & 2.458 & $>1.610^{25}[54]$ & $1.45 E-14$ & $2.19-4.20$ & $<[0.25-0.48]$ \\
\hline${ }^{150} \mathrm{Nd}$ & 3.371 & $>1.810^{22}[55]$ & $6.19 E-14$ & $1.71-3.16$ & $<[4.84-8.95]$ \\
\hline
\end{tabular}

isotopes are already exploring the quasi-degenerate scenarios for the neutrino mass hierarchy (which is around $0.5 \mathrm{eV}$ ). With the ingredients presented in Table 2 (PSFs and NMEs) one can appreciate, as well, the sensitivities, translated into neutrino mass parameters, of the future generation of DBD experiments.

\section{Conclusions}

We report new values of light Majorana neutrino mass parameters from a $0 \nu \beta \beta$ decay analysis extended to all the isotopes for which theoretical and experimental data exists. We used the most recent results for the experimental lifetime $T_{1 / 2}^{0 \nu}$ as well as for the theoretical quantities $G^{0 \nu}$ and $M^{0 \nu}$. For the PSFs we use newly obtained values, recalculated with an approach described in [41] but with improved numerical accuracy. We use exact electron w.f. obtained by solving a Dirac equation when finite nuclear size and screening effects are included and, in addition, a Coulomb potential derived from a realistic proton distribution in the daughter nucleus has been employed. For choosing the NMEs we take advantage of the general consensus in the community on several nuclear ingredients involved in the calculations (HOC, FHS, and SRCs effects, values of several nuclear input parameters) and restrict the range of spread of the NMEs values, reported in the literature. This, in turn, reduces the uncertainty in deriving constraints on the light Majorana neutrino mass parameters, while taking into account NME values obtained with all the main nuclear methods. The stringent constraints are obtained from the ${ }^{136} \mathrm{Xe}$ and ${ }^{76} \mathrm{Ge}$ isotopes, due to both the experimental sensitivity and the reliability of the PSFs and NMEs calculations. The experiments measuring these isotopes are already exploring the quasi-degenerate scenarios for the neutrino mass hierarchy which is around $0.5 \mathrm{eV}$. Our results may be useful for having an up-to-date image on the current neutrino mass sensitivities associated with $0 \nu \beta \beta$ measurements for different isotopes and to better estimate the range of the neutrino masses that can be explored in the future DBD experiments.

Note. After the submission of this paper Exo-2000 published their 2-year new limits for the neutrinoless double beta decay of ${ }^{136} \mathrm{Xe}$, which is less than the value that we used for deriving the neutrino mass parameter in this case. For this isotope, the presently stringent limit for the lifetime is $1.9 \times 10^{25} \mathrm{y}$, from KamLand-Zen experiment [33]. Hence, we use this value, which shifts the interval of the neutrino mass parameter to [0.23-0.44], which represents a small change in our results for this isotope.

\section{Conflict of Interests}

The authors declare that there is no conflict of interests regarding the publication of this paper. 


\section{Acknowledgments}

This work was done with the support of the MEN and UEFISCDI through the project IDEI-PCE-3-1318, Contract no. 58/28.10/2011, and Project PN-09-37-01-02/2009.

\section{References}

[1] F. T. Avignone, S. R. Elliott, and J. Engel, "Double beta decay, Majorana neutrinos, and neutrino mass," Reviews of Modern Physics, vol. 80, no. 2, pp. 481-516, 2008.

[2] P. Vogel, "Nuclear structure and double beta decay," Journal of Physics G: Nuclear and Particle Physics, vol. 39, no. 12, Article ID 124002, 2012.

[3] A. Faessler, V. Rodin, and F. Simkovic, "Nuclear matrix elements for neutrinoless double-beta decay and double-electron capture," Journal of Physics G: Nuclear and Particle Physics, vol. 39, no. 12, Article ID 124006, 2012.

[4] J. Vergados, H. Ejiri, and F. Simkovic, "Theory of neutrinoless double-beta decay," Reports on Progress in Physics, vol. 75, no. 10, Article ID 106301, 2012.

[5] H. Ejiri, "Double beta decays and neutrino nuclear responses," Progress in Particle and Nuclear Physics, vol. 4, no. 2, pp. 249257, 2010.

[6] W. Rodejohann, "Neutrinoless double-beta decay and neutrino physics," Journal of Physics G: Nuclear and Particle Physics, vol. 39, no. 12, Article ID 124008, 2012.

[7] F. Kaether, W. Hampel, G. Heusser, J. Kiko, and T. Kirsten, "Reanalysis of the Gallex solar neutrino flux and source experiments," Physics Letters B, vol. 685, no. 1, pp. 47-54, 2010.

[8] A. Gando, Y. Gando, K. Ichimura et al., "Constraints on $\theta_{13}$ from a three-flavor oscillation analysis of reactor antineutrinos at KamLAND," Physical Review D, vol. 83, Article ID 052002, 2011.

[9] K. Abe, Y. Hayato, T. Iida et al., "Solar neutrino results in SuperKamiokande-III," Physical Review D, vol. 83, Article ID 052010, 2010.

[10] Y. Abe, C. Aberle, T. Akiri et al., "Indication of reactor $\bar{\nu}_{e}$ disappearance in the double chooz experiment," Physical Review Letters, vol. 108, Article ID 131801, 2012.

[11] F. P. An, J. Z. Bai, A. B. Balantekin et al., "Observation of electron-antineutrino disappearance at daya bay," Physical Review Letters, vol. 108, Article ID 171803, 2012.

[12] B. Aharmim, S. N. Ahmed, A. E. Anthony et al., "Low-energythreshold analysis of the phase I and phase II data sets of the Sudbury Neutrino Observatory," vol. 81, Article ID 055504, 2010.

[13] V. A. Rodin, A. Faessler, F. Simkovic, and P. Vogel, "Uncertainty in the $0 \nu \beta \beta$ decay nuclear matrix elements," Physical Review C, vol. 68, Article ID 044302, 2003.

[14] V. A. Rodin, A. Faessler, F. Simkovic, and P. Vogel, "Erratum to: "Assessment of uncertainties in QRPA $0 \nu \beta \beta$-decay nuclear matrix elements" [Nucl. Phys. A 766 (2006) 107]," Nuclear Physics A, vol. 793, no. 1-4, pp. 213-215, 2007.

[15] S. Stoica and H. V. Klapdor-Kleingrothaus, "Critical view on double-beta decay matrix elements within Quasi Random Phase Approximation-based methods," Nuclear Physics A, vol. 694, no. 1-2, pp. 269-294, 2001.

[16] F. Simkovic, A. Faessler, H. Muther, V. Rodin, and M. Stauf, " $0 \nu \beta \beta$-decay nuclear matrix elements with self-consistent short-range correlations," Physical Review C, vol. 79, Article ID 055501, 2009.

[17] M. Kortelainen, O. Civitarese, J. Suhonen, and J. Toivanen, "Short-range correlations and neutrinoless double beta decay," Physics Letters B, vol. 647, no. 2-3, pp. 128-132, 2007.

[18] M. Kortelainen and J. Suhonen, "Improved short-range correlations and $0 \nu \beta \beta$ nuclear matrix elements of ${ }^{76} \mathrm{Ge}$ and ${ }^{82} \mathrm{Se}$," Physical Review C, vol. 75, Article ID 051303, 2007.

[19] F. Simkovic, A. Faessler, V. Rodin, P. Vogel, and J. Engel, "Anatomy of the $0 \nu \beta \beta$ nuclear matrix elements," Physical Review C, vol. 77, Article ID 045503, 2008.

[20] M. Kortelainen and J. Suhonen (Jyvaskyla U.), "Nuclear matrix elements of neutrinoless double beta decay with improved short-range correlations," Physical Review C, vol. 76, Article ID 024315, 2007.

[21] E. Caurier, A. P. Zuker, A. Poves, and G. Martinez-Pinedo, "Full pf shell model study of $A=48$ nuclei," Physical Review $C$, vol. 50, p. 225, 1994.

[22] J. Retamosa, E. Caurier, and F. Nowacki, "Neutrinoless double beta decay of ${ }^{48}$ Ca," Physical Review C, vol. 51, p. 371, 1995.

[23] M. Horoi and S. Stoica, "Shell model analysis of the neutrinoless double- $\beta$ decay of ${ }^{48}$ Ca," Physical Review $C$, vol. 81, Article ID 024321, 2010.

[24] J. Menéndez, A. Poves, E. Caurier, and F. Nowacki, "Disassembling the nuclear matrix elements of the neutrinoless $\beta \beta$ decay," Nuclear Physics A, vol. 818, no. 3-4, pp. 139-151, 2009.

[25] E. Caurier, J. Menendez, F. Nowacki, and A. Poves, "Influence of pairing on the nuclear matrix elements of the neutrinoless $\beta \beta$ decays," Physical Review Letters, vol. 100, Article ID 052503, 2008.

[26] J. Barea and F. Iachello, "Neutrinoless double- $\beta$ decay in the microscopic interacting boson model," Physical Review C, vol. 79, Article ID 044301, 2009.

[27] J. Barea, J. Kotila, and F. Iachello, "Nuclear matrix elements for double- $\beta$ decay," Physical Review C, vol. 87, Article ID 014315, 2013.

[28] J. Barea, J. Kotila, and F. Iachello, "Limits on neutrino masses from neutrinoless double- $\beta$ decay," Physical Review Letters, vol. 109, Article ID 042501, 2012.

[29] P. K. Rath, R. Chandra, K. Chaturvedi, P. K. Raina, and J. G. Hirsch, "Uncertainties in nuclear transition matrix elements for neutrinoless $\beta \beta$ decay within the projected-Hartree-FockBogoliubov model," Physical Review C, vol. 82, Article ID 064310, 2010.

[30] P. K. Rath, R. Chandra, K. Chaturvedi, P. Lohani, P. K. Raina, and J. G. Hirsch, "Neutrinoless $\beta \beta$ decay transition matrix elements within mechanisms involving light Majorana neutrinos, classical Majorons, and sterile neutrinos," Physical Review C, vol. 88, Article ID 064322, 2013.

[31] T. R. Rodriguez and G. Martinez-Pinedo, "Energy density functional study of nuclear matrix elements for neutrinoless $\beta \beta$ decay," Physical Review Letters, vol. 105, Article ID 252503, 2010.

[32] H. Primakov and S. P. Rosen, "Double beta decay," Reports on Progress in Physics, vol. 22, p. 121, 1959.

[33] J. Suhonen and O. Civitarese, "Weak-interaction and nuclearstructure aspects of nuclear double beta decay," Physics Report, vol. 300, no. 3-4, pp. 123-214, 1998.

[34] T. Tomoda, "Double beta decay," Reports on Progress in Physics, vol. 54, no. 1, p. 53, 1991.

[35] M. Doi, T. Kotani, H. Nishiura, and E. Takasugi, "Double beta decay," Progress of Theoretical Physics, vol. 69, no. 2, pp. 602-635, 1983. 
[36] M. Doi and T. Kotani, "Neutrino emitting modes of double beta decay," Progress of Theoretical Physics, vol. 87, no. 5, pp. 12071231, 1992.

[37] M. Doi and T. Kotani, "Neutrinoless modes of double beta decay," Progress of Theoretical Physics, vol. 89, no. 1, pp. 139-159, 1993.

[38] W. C. Haxton and G. J. Stephenson Jr., "Double beta decay," Progress in Particle and Nuclear Physics, vol. 12, pp. 409-479, 1984.

[39] M. Doi, T. Kotani, and E. Takasugi, "Double beta decay and majorana neutrino," Progress of Theoretical Physics Supplements, vol. 83, pp. 1-175, 1985.

[40] J. Kotila and F. Iachello, "Phase-space factors for double- $\beta$ decay," Physical Review C, vol. 85, Article ID 034316, 2012.

[41] S. Stoica and M. Mirea, "New calculations for phase space factors involved in double- $\beta$ decay," Physical Review $C$, vol. 88, Article ID 037303, 2013.

[42] T. E. Pahomi, A. Neacsu, M. Mirea, and S. Stoica, "Phase space calculations for beta- beta- decays to final excited $2+1$ states," Romanian Reports in Physics, vol. 66, 2, p. 370, 2014.

[43] A. Neacsu and S. Stoica, "Study of nuclear effects in the computation of the $0 v \beta \beta$ decay matrix elements," Journal of Physics G: Nuclear and Particle Physics, vol. 41, no. 1, Article ID 015201, 2014.

[44] M. Horoi, "Shell model analysis of competing contributions to the double- $\beta$ decay of ${ }^{48}$ Ca," Physical Review $C$, vol. 87, Article ID 014320, 2013.

[45] M. Horoi and B. A. Brown, "Shell-model analysis of the ${ }^{136} \mathrm{Xe}$ double beta decay nuclear matrix elements," Physical Review Letters, vol. 110, Article ID 222502, 2013.

[46] D. L. Fang A Faessler, V. Rodin, and F. Šimkovic, "Neutrinoless double- $\beta$ decay of ${ }^{150} \mathrm{Nd}$ accounting for deformation," Physical Review C, vol. 82, Article ID 051301(R), 2010.

[47] O. Civitarese and J. Suhonen, "Nuclear matrix elements for double beta decay in the QRPA approach: a critical review," Journal of Physics: Conference Series, vol. 173, no. 1, Article ID 012012, 2009.

[48] S. Umehara, T. Kishimoto, I. Ogawa et al., "Neutrino-less double- $\beta$ decay of ${ }^{48} \mathrm{Ca}$ studied by $\mathrm{CaF}_{2}(\mathrm{Eu})$ scintillators," Physical Review C, vol. 78, Article ID 058501, 2008.

[49] C. Macolino and GERDA Collaboration, "Results on neutrinoless double-beta decay from GERDA phase I," Modern Physics Letters A, vol. 29, no. 1, Article ID 1430001, 20 pages, 2014.

[50] A. S. Barabash and V. B. Brudanin, "Investigation of double-beta decay with the NEMO-3 detector," Physics of Atomic Nuclei, vol. 74, no. 2, pp. 312-317, 2011.

[51] J. Argyriades, R. Arnold, C. Augier et al., "Measurement of the two neutrino double beta decay half-life of $\mathrm{Zr}-96$ with the NEMO-3 detector," Nuclear Physics A, vol. 847, no. 3-4, pp. 168$179,2010$.

[52] F. A. Danevich, A. S. Georgadze, V. V. Kobychev et al., "Search for $2 \beta$ decay of cadmium and tungsten isotopes: final results of the Solotvina experiment," Physical Review C, vol. 68, Article ID 035501, 2003.

[53] E. Andreotti, C. Arnaboldi, and F.T. Avignone III, “130 Te neutrinoless double-beta decay with CUORICINO," Astroparticle Physics, vol. 34, no. 11, pp. 822-831, 2011.

[54] A. Gando, Y. Gando, H. Hanakago et al., "Limit on neutrinoless $\beta \beta$ decay of ${ }^{136} \mathrm{Xe}$ from the first phase of KamLAND-Zen and comparison with the positive claim in ${ }^{76} \mathrm{Ge}$," Physical Review Letters, vol. 110, Article ID 062502, 2013.
[55] J. Argyriades, R. Arnold, C. Augier et al., "Measurement of the double- $\beta$ decay half-life of Nd150 and search for neutrinoless decay modes with the NEMO-3 detector," Physical Review C, vol. 80, Article ID 032501(R), 2009.

[56] S. F. King and C. Luhn, "Neutrino mass and mixing with discrete symmetry," Reports on Progress in Physics, vol. 76, no. 5, Article ID 056201, 2013.

[57] A. Giuliani and A. Poves, "Neutrinoless double-beta decay," Advances in High Energy Physics, vol. 2012, Article ID 857016, 38 pages, 2012.

[58] C. Giusti, H. Muther, F. D. Pacati, and M. Stauf, "Short-range and tensor correlations in the ${ }^{16} \mathrm{O}\left(\mathrm{e}, \mathrm{e}^{\prime} \mathrm{pn}\right)$ reaction," Physical Review C, vol. 60, Article ID 054608, 1999.

[59] H. Muther A Polls, "Correlations derived from modern nucleon-nucleon potentials," Physical Review C, vol. 61, Article ID 014304, 1999.

[60] H. Müther, "Two-body correlations in nuclear systems," Progress in Particle and Nuclear Physics, vol. 45, no. 1, pp. 243334, 2000.

[61] J. P. Schiffer, S. J. Freeman, J. A. Clark et al., "Nuclear structure relevant to neutrinoless double $\beta$ decay: ${ }^{76} \mathrm{Ge}$ and ${ }^{76} \mathrm{Se}$," Physical Review Letters, vol. 100, Article ID 112501, 2008.

[62] B. P. Kay, J. P. Schiffer, S. J. Freeman et al., "Nuclear structure relevant to neutrinoless double $\beta$ decay: the valence protons in ${ }^{76}$ Ge and ${ }^{76}$ Se," Physical Review C, vol. 79, Article ID 021301(R), 2009.

[63] J. Liu, M. P. Mendenhall, A. T. Holley et al., "Determination of the axial-vector weak coupling constant with ultracold neutrons," Physical Review Letters, vol. 105, Article ID 181803, 2010.

[64] J. Menendez, A. Poves, E. Caurier, and F. Nowacki, "Occupancies of individual orbits, and the nuclear matrix element of the ${ }^{76}$ Ge neutrinoless $\beta \beta$ decay," Physical Review $C$, vol. 80, Article ID 048501, 2009.

[65] E. Caurier, A. Poves, and A. P. Zuker, "A full View 0 h $\omega$ description of the $2 \nu \beta \beta$ decay of ${ }^{48} \mathrm{Ca}$," Physics Letters $B$, vol. 252, no. 1, pp. 13-17, 1990. 

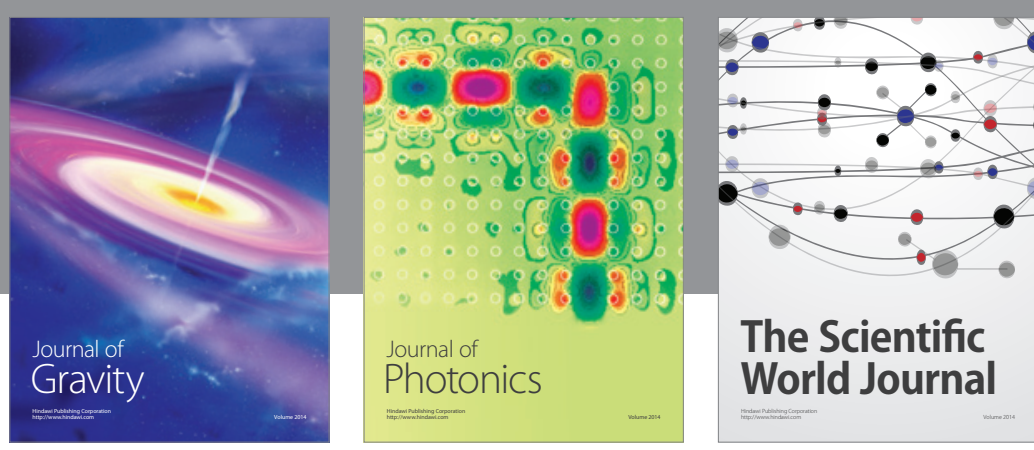

The Scientific World Journal
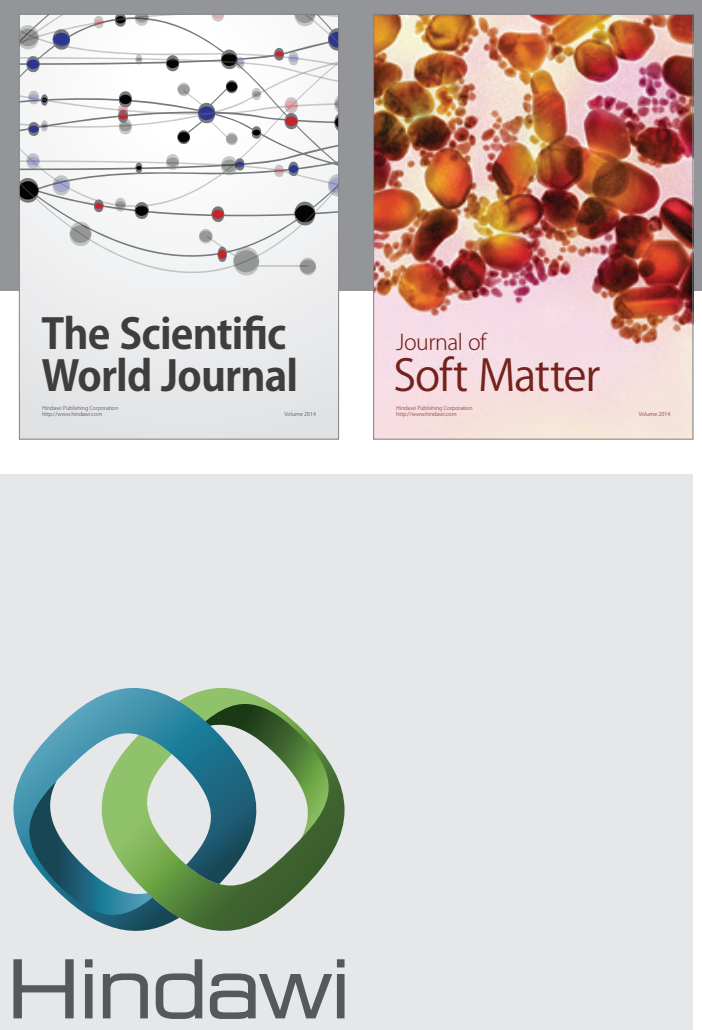

Submit your manuscripts at

http://www.hindawi.com

nternational Journal of

Statistical Mechanics
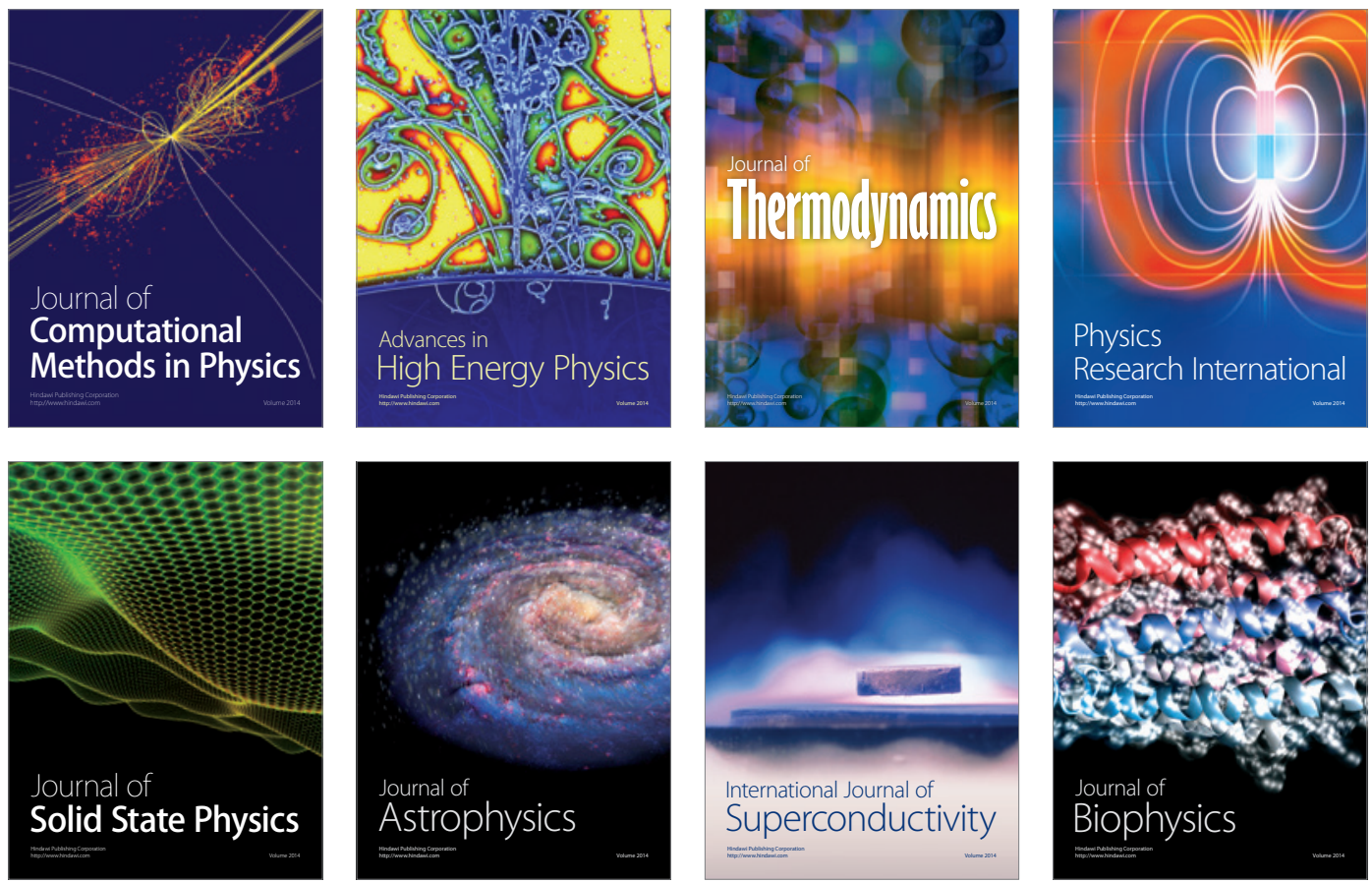
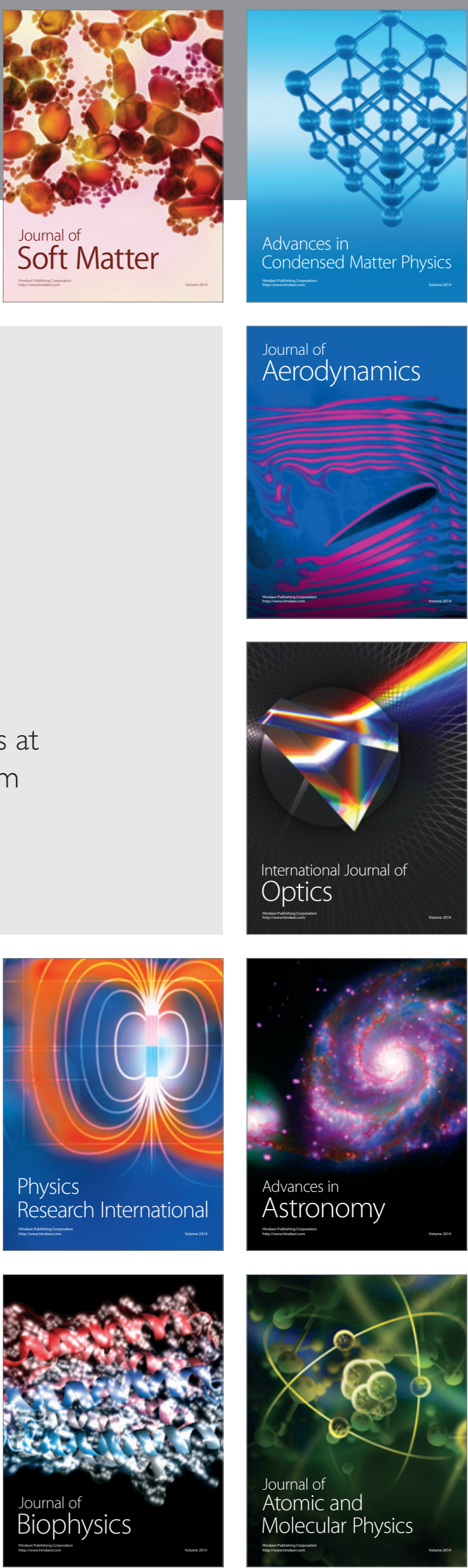